\title{
Fatigue Analysis of Hinged Support Structures for Overhead Conductor Rails based on Power Density Theory
}

\author{
Zhan Chen ${ }^{1}$, Jiqin $\mathrm{Wu}^{1}$, Lei Gan${ }^{1}$, Jianghai $\mathrm{He}^{2}$ and Xiaojun $\mathrm{Li}^{1}$ \\ ${ }^{1}$ School of Electrical Engineering, Southwest Jiaotong University, Chengdu 611756, China \\ ${ }^{2}$ Guangzhou Metro Group Co., Ltd., Guangzhou 510000, China
}

Received 11 February 2020; Accepted 29 May 2020

\begin{abstract}
Hinged support structures are important support components of overhead conductor rails. In practice, hinged support structures are subjected to complex and variable loads when the dynamic contact forces between the pantographs and the overhead conductor rails changes. Hence, they may suffer from great fatigue damage. A novel method for analyzing the influence of load frequency on fatigue life was proposed to predict the fatigue life of hinged support structures for overhead conductor rails under complex loads. The method was used to estimate the fatigue life of a hinged support structure. The material fatigue curve and theory of short-time Fourier transform were combined, and power density was connected to the material fatigue. The influence of load frequency on fatigue life was discussed in depth. A test was carried out to collect the strain-time history at the dangerous point of the hinged support structure, and the time course of the stress was obtained in accordance with the stress-strain relationship. The fatigue life of the hinged support structure was predicted using the proposed method. Results demonstrate that the fatigue life of each test point of the hinged support structure is between 528.26 and $970.09 \mathrm{~h}$. This range of the designed working life is close to 2 million sorties that of the pantograph, that is, an actual working life of $833 \mathrm{~h}$. The proposed method provides a reference for the design and performance evaluation of overhead conductor rails.
\end{abstract}

Keywords: overhead conductor rail; hinged support structure; power density; fatigue damage; fatigue life

\section{Introduction}

Contact line systems serve as the main structure of electric railways. They consist of foundations, support structures, cross-span equipment, traction power lines, disconnectors, and accessories. A train obtains electric energy through the sliding contact between the pantograph and the contact line. The contact line is divided into the overhead conductor rail and the overhead contact line. The overhead conductor rail is mostly used in urban rail transit. Its support structure is an important component that carries almost its entire weight. However, hinged support structures require a high level of voltage and a long power supply arm, particularly for highspeed trains. In China, the Xinguanjiao Tunnel was completed and opened to traffic at the end of 2014; it uses a $25 \mathrm{kV} \mathrm{AC}$ overhead conductor rail with a design speed of $160 \mathrm{~km} / \mathrm{h}$. Guangzhou Metro Line 18 and Beijing New Airport Line have all been planned and constructed with AC $25 \mathrm{kV}$ overhead conductor rails with a maximum operating speed of $160 \mathrm{~km} / \mathrm{h}$. In Austria, a $15 \mathrm{kV}$ AC overhead conductor rail was used in the Sittenberg Tunnel on the Wien-Linz route; the project is the first in Europe to comply with the high-speed infrastructure TSI (Technical Specification for Interoperability) for speeds of $250 \mathrm{~km} / \mathrm{h}$ or higher [1].

With the acceleration of urbanization, urban and suburban areas have become closely connected. Trains in particular have considerably shortened travel times.

*E-mail address: cz953664182@163.com

ISSN: $1791-2377 @ 2020$ School of Science, HUU. All rights reserved.

doi:10.25103/jestr.133.24
However, as the speed increases, the contact force between a train's pantograph and contact line changes drastically, and the components of the contact line become subjected to complex and variable loads. Therefore, contact lines should have strong fatigue resistance, in addition to sufficient strength and rigidity.

Scholars have conducted extensive studies on the prediction of the fatigue life of contact line components [24], and most of them have focused on overhead contact lines. Hence, overhead conductor rails have been rarely studied. The accurate prediction of the fatigue life of the components of overhead conductor rails thus requires immediate attention.

Therefore, a novel method is proposed in the current work to determine the effects of load frequency on fatigue life. The fatigue life of a hinged support structure is predicted to provide a reference for the fatigue prediction of overhead conductor rails.

\section{State of the art}

In the field of rail transit, scholars have conducted comprehensive studies on contact lines. Massat et al. [5] conducted a preliminary analysis of the parameters affecting the fatigue phenomenon of a contact wire by comparing failures that occurred in Japan and France; they also performed a fatigue assessment of the contact wire, but they did not explore its fatigue life. Ritzberger et al. [6] proposed a new method for the dynamic simulation of the contact between pantographs and overhead contact lines, but they did not analyze the impact of the contact force. Bucca et al. 
[7] proposed a heuristic wear model for contact wires subjected to the sliding contact between pantographs and overhead contact lines; although they estimated the amount of wear associated with the interaction between pantographs and overhead contact lines, they did not conduct a fatigue analysis. Kim et al. [8, 9] calculated the cumulative fatigue damage on a $\mathrm{Cu}-\mathrm{Mg}$ contact wire developed for a $400 \mathrm{~km} / \mathrm{h}$ level railway system and investigated the fatigue safety factor on the $\mathrm{Cu}-\mathrm{Mg}$ contact wire at an allowable strain condition; however, their method is unsuitable for overhead conductor rails. Van et al. [10] combined the mass drop test, analysis model, and parametric finite element model simulation and carried out a detailed analysis of the dynamic characteristics of pantographs and overhead contact lines; however, they did not analyze the dynamic characteristics between pantographs and overhead conductor rails. Qi et al. $[4,11]$ compiled the fatigue load spectra of the key parts of a high-speed railway contact line, including the catenary wire support clamp, top anchor, cantilever tube, and diagonal tube, and then carried out a life assessment; however, the individual components were not observed and analyzed in detail. Gregori et al. [12] proposed a fast simulation method for the dynamic interaction between pantographs and overhead contact lines; although this method significantly reduces the computational cost without limiting accuracy and was successfully implemented in efficient optimizations, it is not applicable to the simulation between a pantograph and an overhead conductor rail. Deqiang et al. [13, 14] used the method of tribological analysis and analyzed the failure of the positioning hook and positioning support on the basis of material and service conditions; however, they did not conduct a fatigue analysis. Zhao [15] studied the influence of speed, process and pantograph parameters, and overhead contact line on the fatigue life of a dropper by analyzing the dynamic characteristics of the dropper when the pantograph passes by; the author also predicted the safe driving time for the dropper. However, due to the particularity of the dropper, the method is not generally applicable to other parts. Chen et al. [16] proposed an analytic vibration equation for the fatigue analysis of a dropper and studied the influence of excitation frequency, vibration amplitude, and applied force on the fatigue life of the dropper; however, they did not experimentally verify the results. Zhang et al. [17] proposed a simulation method that considers horizontal vibrations for droppers and reproduced the horizontal vibration of a dropper on site via a laboratory test and computer simulation; however, they did not conduct further analysis of the fatigue life of the dropper. Wei et al. [18] established a calculation model for wear rate that can be used to approximately predict the wear of contact wires and pantograph strips; however, they did not cover fatigue life in their work.

In the aspect of fatigue analysis theory, the traditional fatigue analysis approach, including the nominal stress approaches and local stress-strain approaches [19-21], is mainly based on the Palmgren-Miner linear damage rule. Fomichev et al. Hrabowski et al. [21] described the lowcycle fatigue behavior of modern high-strength and ultrahigh-strength steel with nominal strengths of up to 1100 $\mathrm{N} / \mathrm{mm}^{2}$ in welded conditions; they also defined the application limit of elastic design rules on the basis of nominal stress and the notch stress approach. [22] proposed a fatigue life prediction method for structural elements with material precompression in the whole vicinity from a local stress-strain state that is based on the energy criterion of a fatigue fracture. Fricke et al. [23] used the nominal and structural stress approaches to analyze the fatigue strength of a thin-plated ship structure. However, these approaches do not consider the influence of the frequency of material loading on fatigue life.

The results in the field of rail transit mainly focus on overhead contact lines. Hence, the study on the fatigue life of overhead conductor rails, especially the study on the correlation of the fatigue lives of overhead conductor rail support structures, is limited. In addition, the traditional fatigue analysis approaches are mainly based on the nominal stress approaches and local stress-strain approaches, neither of which considers load frequency. The present study proposes a novel method for analyzing the fatigue life of hinged support structures. In this study, the influence of amplitude and frequency is discussed in depth. The material fatigue curves and the short-time Fourier transform (STFT) theory are combined, and power density is related to material fatigue. A test for the collection of dynamic stress is carried out, and fatigue life is predicted by the proposed method. The results should provide a reference for the fatigue prediction of overhead conductor rails.

The remainder of this study is organized as follows. Section 3 describes the theoretical basis of the proposed method, that is, power density theory. It also presents the process of the test for the collection of the dynamic stress of a hinged support structure. Section 4 details the fatigue life predicted by the power density method on the basis of the test data of the hinged support structure. Section 5 summarizes this study and provides several conclusions.

\section{Methodology}

\subsection{Power density theory}

The stress wave borne by the material is defined as $\sigma(t)$. The effect of stress on the material in unit time, that is, the gradient of stress on time $(d \sigma(t) / d t)$, is investigated. From the unit point of view, $\left(\mathrm{N} / \mathrm{m}^{2}\right) \cdot(1 / \mathrm{s})=\mathrm{N} / \mathrm{m} /\left(\mathrm{s} \cdot \mathrm{m}^{2}\right)=\mathrm{W} / \mathrm{m}^{3}$ is used to identify that the gradient of stress on time is the power generated by the load on the material in unit volume; such power is defined as the power density. Assuming that the stress signal is $\sigma(t)=A \sin (2 \pi f t)$, the power density can be expressed as $d \sigma(t) / d t=A \cdot 2 \pi f \cdot \cos (2 \pi f)$. The fatigue analysis method can also reflect the influence of load amplitude $(A)$ and frequency $(f)$.

The definition of power density indicates that the effect of stress on the material is only related to three factors, namely, stress amplitude ( $A$ ), load frequency $(f)$, and duration of load $(t)$. According to the characteristics of materials, power density theory has the following characteristics:

1) When the power density of a material under a certain load reaches or exceeds its rated allowable power density, the material cracks under this load. The cracks that form then expand;

2) Under a certain load, no cracks form and expand when the power density does not reach the allowable value;

3) When the load exceeds the allowable power density, the cumulative effect of power density causes the cracks of the material to continue to expand. The accumulation of energy generated during the period from the generation of cracks to the failure of materials is called the ultimate accumulated energy. 


\subsection{Linear fatigue damage accumulation}

The hypothesis of linear fatigue damage accumulation is as follows:

1) During the load process of the structure, each load cycle causes certain damage;

2) Fatigue damage is proportional to the energy that the load acts on;

3) When the damage value $(D)$ reaches a certain value, fatigue damage or failure of the structure occurs ( $D$ is generally used to indicate the structural damage rate; the failure criterion is $D=1$ ).

Under variable amplitude loads, the total damage is the sum of the damage values under a single amplitude load; that is,

$$
D=\sum_{i=1}^{k} D_{i}
$$

where $k$ is the number of stress levels of the variable amplitude load, that is, the total number of different amplitudes; $D_{i}$ is the damage caused by the $i$-th amplitude load. The reciprocal of the fatigue damage value is the serviceable times of the structure, that is, the fatigue life of the structure given by

$$
N=1 / D
$$

\subsection{Time-frequency analysis method}

The STFT method is used in the time-frequency analysis, and the signal is processed by a certain window function. The local time-frequency analysis of the whole signal is realized by Fourier transform of the segmented signal. The nonstationary signal is regarded as a series of short-time stationary signal superposition, and the short time is realized by the window function. The whole time domain is covered by the parameter translation. The mathematical expression of STFT is as follows:

$$
T\left(w_{n}, b_{m}\right)=\int_{-\infty}^{+\infty} f(t) \cdot g\left(t-b_{m}\right) e^{-i w_{n} t} d t
$$

where $T\left(w_{n}, b_{m}\right)$ is the spectrum; $f(t)$ is the original time domain signal; $g\left(t-b_{m}\right)$ is the window function; $w_{n}$ is the window frequency domain width, the unit of which is $\mathrm{Hz}$; $b_{m}$ is the window time domain width, the unit of which is $\mathrm{s}$; $t$ is the time, the unit of which is $\mathrm{s} ; i$ is the imaginary unit. The discrete form of the above formula is as follows:

$$
T\left(w_{n}, b_{m}\right)=\sum_{n=0}^{m} f(n \Delta t) g\left(n \Delta t-b_{m}\right) e^{-i w_{n} t} \Delta t
$$

where $m$ is the total number of time steps; $n$ is the time step, $n=1,2, \ldots, m ; \Delta t$ is the width of the window function, the unit of which is $\mathrm{s} ; n \Delta t(n=1,2, \ldots, m)$ represents the time of each signal point, the unit of which is $\mathrm{s}$.

\subsection{Fatigue analysis method}

In the case of the known stress-life curve of materials, the equation of the power density-life curve can be obtained by the gradient of time as:

$$
d S / d t=\operatorname{function} 1\left(N_{f}\right)
$$

where $N_{f}$ is the number of limit cycles; $d S / d t$ is the power density of the load, the unit of which is $\mathrm{W} / \mathrm{m}^{3}$. The inverse function can be obtained as follows:

$$
N_{f}=\text { function } 2(d S / d t)
$$

For broadband signals, at any time $\left(t=t_{0}\right)$, the power density $\left((d S / d t)_{t=t_{0}}\right)$ can be decomposed into a number of cosine signals related to each main frequency $\left(f_{i}\right)$ and power density $\left(A_{i}=(d S / d t)_{t=t_{0}}\right)$ by STFT:

$$
(d S / d t)_{t=t_{0}}=\sum_{i} A_{i} \cos \left(F_{i} \cdot \Delta t\right)
$$

In the time period ( $\Delta t)$ of time $\left(t=t_{0}\right)$, the damage rate caused by the power density ( $(d S / d t)_{t=t_{0}}$ ) is as follows:

$$
D=\sum_{i} N_{i} /\left(N_{f}\right)_{i}
$$

where $D$ is the damage rate in time period ( $\Delta t)$; $\left(N_{f}\right)_{i}=f 2\left[(d S / d t)_{t=t_{0, i}}\right],\left(N_{f}\right)_{i}$ is the number of limit cycles of material fatigue failure corresponding to the main frequency $\left(F_{i}\right) ; \quad N_{i}=F_{i} \Delta t, N_{i}$ is the number of cumulative cycles in the time interval of $\Delta t$ at $t=t_{0}$ corresponding to the main frequency $\left(F_{i}\right)$.

In a given time step $m$, the cumulative damage rate of the material is as follows:

$$
A D=\sum_{j=1}^{m}\left(\sum_{i} N_{j, i} /\left(N_{f}\right)_{j, i}\right)
$$

where $A D$ is the cumulative damage rate of the material; and $j$ is the given time step, $j=1,2, \ldots, m$. The failure criterion of the material can be obtained as follows:

$$
A D=\sum_{j=1}^{m}\left(\sum_{i} N_{j, i} /\left(N_{f}\right)_{j, i}\right) \geq 1
$$

Under the action of typical load, within a certain statistical error range, the fatigue life of materials can be estimated as follows:

$F L=T / A D$

where $F L$ is the estimated fatigue life of the material, the unit of which is $\mathrm{s}$; and $T$ is the time of the typical load action, the unit of which is $\mathrm{s}$.

\subsection{Test for collection of dynamic stress}

\subsubsection{Principle of test}

Strain gauges are stuck on the location of the maximum stress and deformation obtained by the finite element analysis. The dynamic stress-time history of the hinged support structure is used as the input data; it is obtained by 
the dynamic simulation of the pantograph and the overhead conductor rail under different levels of speed. The straintime history at the dangerous point of the hinged support structure is obtained. The time course of the stress can then be obtained according to the stress-strain relationship. On the one hand, the situation of the overall stress and strain of the hinged support structure can be analyzed from the perspective of the test. On the other hand, data support for the subsequent fatigue analysis is provided.

When the strain gauge is pasted on the tested structure according to the specification, its resistance is changed slightly with the deformation of the structure. After the strain gauges are combined into a bridge circuit, the small change of resistance for the strain gauge is transformed into a voltage signal, which is easy to measure. After the voltage signals are amplified by the amplifying circuit and collected by the acquisition card, the stress and strain of the structure can be obtained by the changes of the voltage signals.

This test uses a single-arm bridge, and the principle is shown in Fig. 1.

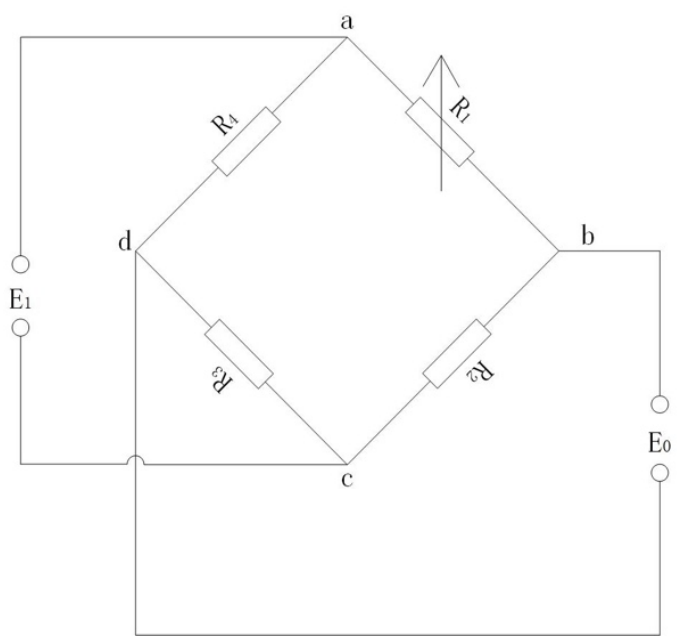

Fig. 1. Test schematic of single-arm bridge

In the figure, $E_{1}$ is the excitation voltage of the bridge, $E_{2}$ is the output voltage signal of the bridge, $R_{1}$ is the strain gauge, and the remaining variables denote the fixed resistance.

$$
E_{0}=\left[\frac{R_{1}}{R_{1}+R_{2}}-\frac{R_{4}}{R_{3}+R_{4}}\right] E_{1}=\frac{R_{1} R_{2}-R_{3} R_{4}}{\left(R_{1}+R_{2}\right)\left(R_{3}+R_{4}\right)} E_{1}
$$

When $R_{1}=R_{2}=R_{3}=R_{4}$, the bridge is a full-arm bridge, and the output voltage is $E_{0}=0$.

When the change in the resistance of $R_{1}$ is $\Delta R$, the output voltage of the bridge is as follows:

$E_{0}=\left[\frac{R_{1}+\Delta R}{R_{1}+R_{2}}-\frac{R_{4}}{R_{3}+R_{4}}\right] E_{1}=\frac{\Delta R}{4 R+2 \Delta R} E_{1}$

where $2 \Delta R$ in the denominator can be ignored because of $\Delta R \ll R$; then:

$E_{0}=\frac{\Delta R}{4 R} E_{1}=\frac{E_{1}}{4} K \varepsilon$ where $\varepsilon$ is the strain and $K$ is the sensitivity coefficient of the strain gauge, which is the intrinsic parameter of the strain gauge generally provided by the manufacturer. According to equation (14):

$$
\varepsilon=\frac{4 E_{0}}{E_{1} K}
$$

On the premise that the excitation voltage $\left(E_{1}\right)$ and sensitivity coefficient $(K)$ are known, the information of the strain can be obtained as long as the output signal $\left(E_{0}\right)$ is collected.

\subsubsection{Testing equipment}

The main materials and equipment used in this test include a strain gauge, a signal amplifier, a data acquisition card, a computer, and an acquisition program. The specific parameters are as follows:

a. Strain gauge: BX120-3AA, resistance strain gauge of acetal from China Aerospace Aerodynamics Research Institute, with a sensitivity coefficient of $2 \pm 1 \%$;

b. Signal amplifier: PARAGON PA-1192, isolated safety barrier of strain bridge; response frequency is $1000 \mathrm{~Hz}$, response precision is $0.1 \%$, input range is $\pm 50 \mathrm{mV}$, output range is $0 \sim 10 \mathrm{~V}$;

c. Data acquisition card: NI USB-6216 acquisition card, multifunctional DAQ module of USB M series from NI company, which can maintain high accuracy under high sampling frequency;

d. Computer and data acquisition program: CVI software.

The signal acquisition scheme of this test according to the testing principle and equipment is shown in Fig.2. According to the operation specification, the strain gauge is pasted on the selected test point, to which the Wheatstone strain bridge is connected. The initial voltage signal can then be obtained after the excitation voltage input. The voltage signal can be output and collected through the module for signal amplification and then stored by the $\mathrm{Ni}$ acquisition card and CVI software. According to the Shannon sampling theorem, the sampling frequency of the signal is $500 \mathrm{~Hz}$, which is more than double the highest analysis frequency.

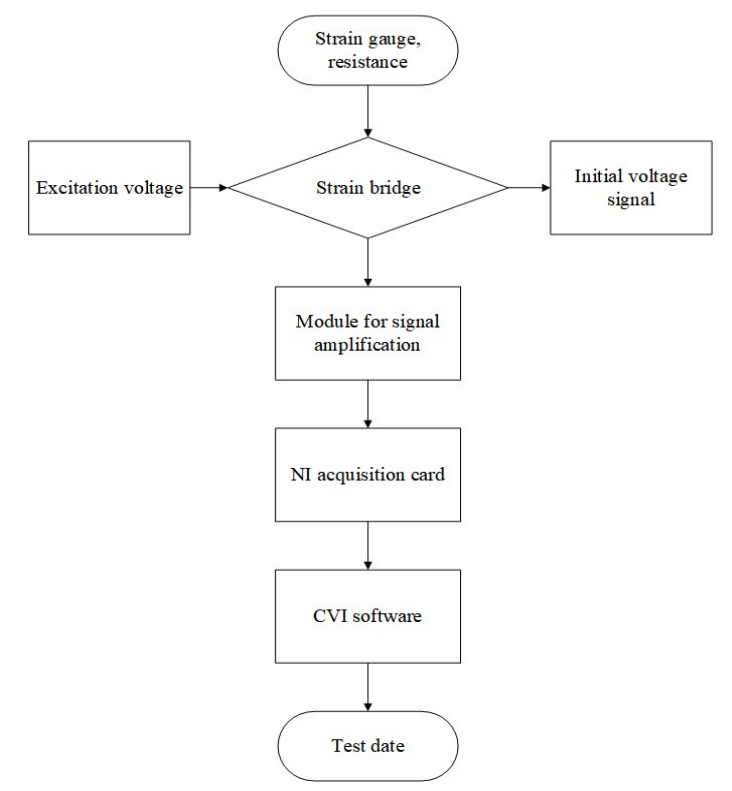

Fig. 2. Schematic of signal acquisition 


\subsubsection{Selection of test points}

When carrying out the test for the collection of dynamic stress, the test point should be selected at the location of maximum stress and deformation. Prior to the test, the failure of the hinged support structure in the actual work of the overhead conductor rail is investigated. Then, the location of the hinged support structure that is most prone to failure in the actual work is determined as the elastic clamp and rotating base. On the basis of the results of the finite element analysis, the location of the maximum stress and deformation on the hinged support structure are selected as the test points. The location of the test points is shown in Fig.

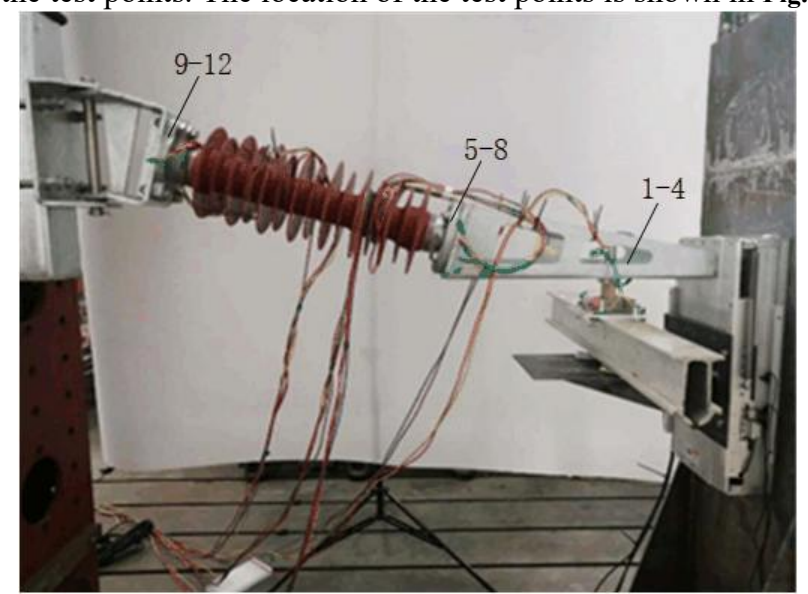

Fig. 3. Location of test point

Four test points are grouped for signal collection. Test points $1-4$ are the positions where the elastic clamp is located with the maximum structural stress; test points 5-8 are the positions between the insulator and the connecting plate of the elastic clamp; test points 9-12 are the positions where the rotating base is located.

\section{Result Analysis and Discussion}

\subsection{Data processing and analysis}

The signal of the output voltage ( $E_{0}$ ) of the strain bridge is basically at the level of $m V$ because the resistance of the strain gauge changes slightly. Amplifying the signal prior to acquisition is necessary to facilitate the acquisition and analysis of signals.

According to the working principle of the signal amplifier, its output signal is as follows:

$$
E_{02}=100 E_{0}+5
$$

where $E_{02}$ is the output voltage of the amplifier, that is, the signal collected by the NI acquisition card.

The initial value of the voltage signal is assumed to be $E_{m}$, and the initial signal collected after being amplified by the amplifier is $E_{m 2}=100 E_{m}+5$. Then, the output voltage signal of the bridge is as follows:

$$
E_{0}^{\prime}=E_{0}-E_{m}=\frac{E_{02}-5}{100}-\frac{E_{m 2}-5}{100}=\frac{E_{02}-E_{m 2}}{100}
$$

Equations (16) and (17) show that:

$$
\varepsilon=\frac{E_{02}-E_{m 2}}{25 E_{i} K}
$$

According to Hooke's law, if the material is in the stage of linear elasticity, that is, when the stress does not exceed the proportional limit of the material, then stress is proportional to stress, that is:

$$
\sigma=E \cdot \varepsilon=E \cdot \frac{E_{02}-E_{m 2}}{25 E_{i} K}
$$

The strain gauge is pasted, and the initial value is collected prior to the assembly of the testing bench to consider the effect of testing load on the hinged support structure under static state.

After the testing bench is built, each test point is initially tested under static conditions. The average stress is shown in Table 1.

Table 1. Average stress of each test point in static state

\begin{tabular}{|l|l|l|l|l|l|l|}
\hline Test print & 1 & 2 & 3 & 4 & 5 & 6 \\
\hline $\begin{array}{l}\text { Average } \\
\text { load /MPa } \\
\text { Test print }\end{array}$ & 103.7 & 104.9 & 112.3 & 114.4 & 86.0 & 85.4 \\
$\begin{array}{l}\text { Average } \\
\text { load /MPa }\end{array}$ & 65.4 & 6 & 9 & 10 & 11 & 12 \\
\hline
\end{tabular}

Table 1 shows that among the test points of the hinged support structure, test points 1 and 2 bear high stress because they are the direct input points of the force at the location of the elastic clamp. Test points 5 and 6 bear high stress as well due to the gravity of the connecting plate of the elastic clamp and the insulator. Test points 9 and 10 are located at the connection part between the rotating base and the davit because the full weight of the hinged support structure is transferred to the davit through this position. These test points on the hinged support structure bear the maximum load.

The counterweight of $94.56 \mathrm{~kg}$ is added to the elastic clamp (the maximum span is $8 \mathrm{~m}, 5.91 \mathrm{~kg}$ per meter), and the testing bench is horizontally placed to simulate the working condition of the hinged support structure in an actual operation. The tool components, such as the davit and fastening bolt, are installed under actual working condition to mimic the field working condition as much as possible and reduce the testing error. The stress-time curve of each test point is shown in Fig. 4.

The main material of the hinged support structure is ordinary carbon structural steel (Q235A), with tensile strength of $\sigma_{b}=500 \mathrm{MPa}$ and yield strength of $\sigma_{s}=235 \mathrm{MPa}$. The schematic of stress-life curve is shown in Fig. 5.

In the figure, $N_{v}$ is the number of equivalent cycles, $\sigma_{r v}$ is the fatigue limit corresponding to $N_{v}, N_{0}$ is the cyclic cardinal number of the material, and $\sigma_{r}$ is the fatigue limit corresponding to $N_{0}$. According to the exponential relationship of the equation of the fatigue curve:

$$
\sigma_{r}^{m} \cdot N_{0}=\sigma_{r v}^{m} \cdot N_{v}=C
$$

where $\sigma_{r}=0.23\left(\sigma_{s}+\sigma_{b}\right), m=9, N_{0}=10^{7}$, and $C$ is a constant; according to the principle of equal total life damage rate, when the cyclic characteristic is $r$, the following can be obtained: 


$$
\sigma_{1}^{m} N_{1}+\sigma_{2}^{m} N_{2}+\sigma_{3}^{m} N_{3}+\ldots+=\sigma_{v}^{m} N_{v}
$$

where $\sigma_{1}, \sigma_{2}, \sigma_{3}, \ldots, \sigma_{n}$ is the maximum stress of each cycle, the unit of which is $\mathrm{Pa} ; N_{1}, N_{2}, N_{3}, \ldots, N_{n}$ is the corresponding cumulative number of cycles.

Take test point 4 as an example. The data of test point 4 is processed by cycle counting. The result is substituted into equation (21), the equivalent stress is determined as $\sigma_{v}=114 \mathrm{MPa}$, and the corresponding number of equivalent cycles is calculated as $N_{v}=1.31 \times 10^{5}$. The corresponding equivalent fatigue limit can be calculated as $N_{v}=1.31 \times 10^{5}$ by equation (20).
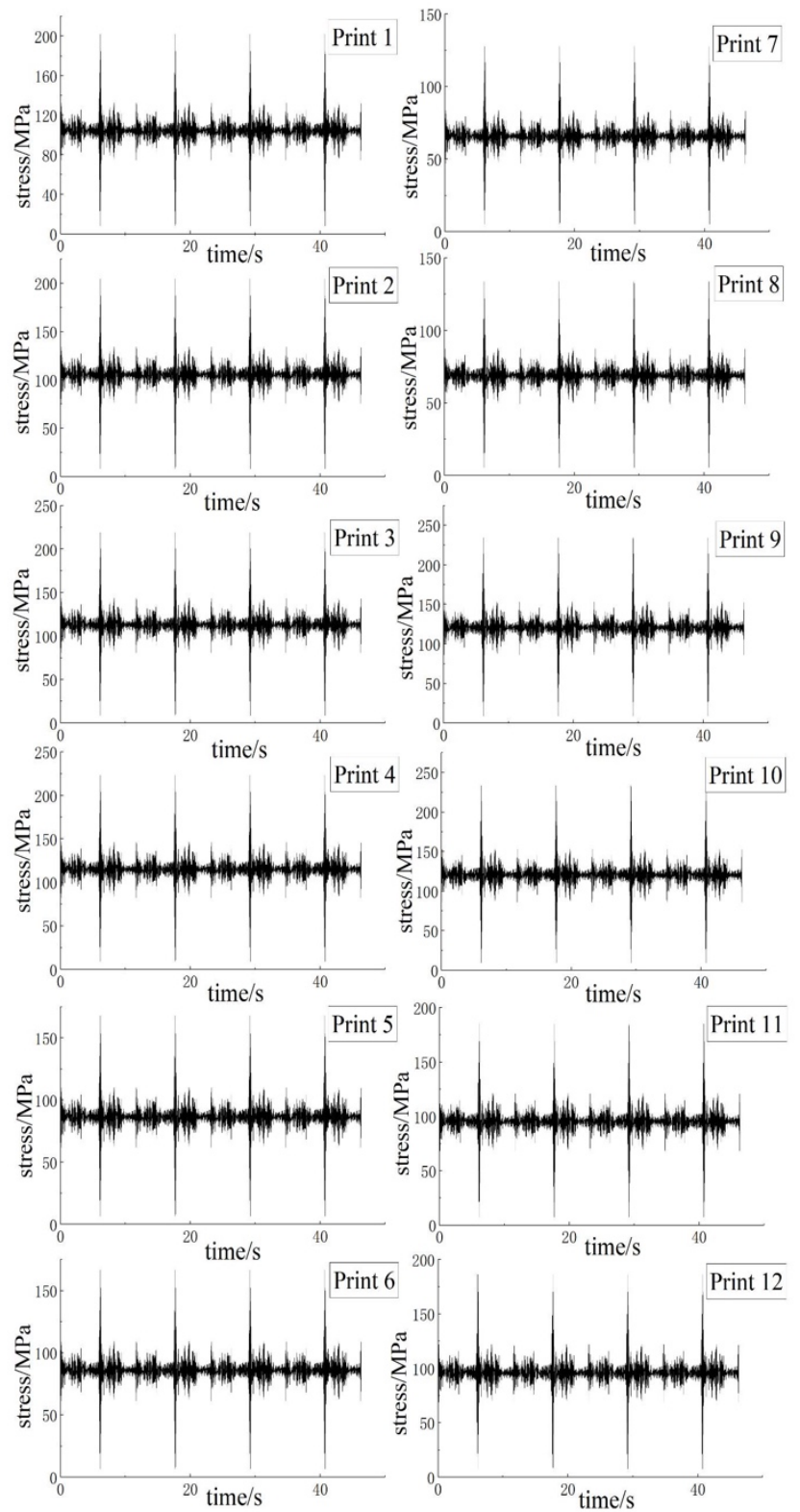

Fig. 4. Stress-time curve of each test point

\subsection{Power density-life characteristics}

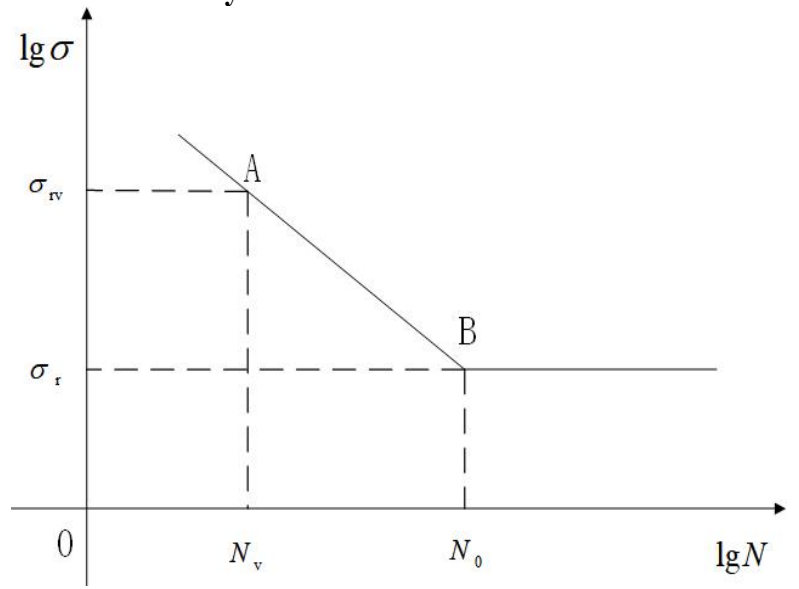

Fig. 5. Stress-life curve diagram

The coordinates of A and B are substituted into Fig. 5, and the expression of the stress-life curve corresponding to the A-B section of the limited fatigue life can be obtained as follows:

$$
S=(656.11-65.30 \cdot \lg N) \times 10^{6}
$$

where $S$ is the stress of fatigue limit, the unit of which is $\mathrm{Pa}$. follows:

The power density-life curve can be expressed as

$d s / d t=K \cdot(656.11-65.30 \lg N) \times 10^{6}$

where $K$ is the absolute value of the ratio of power density to stress at different times. The limit cycles of the hinged support structure can be expressed as functions of $d s / d t$ :

$\left.N=10^{\left[10.05-1.53 \times 10^{-8} \times(d s / d t) / K\right.}\right]$

\subsection{Frequency domain analysis}

In accordance with the concept of power density, the results shown in Fig. 4 are deduced against time, and the power density-time curve of each test point of the hinged support structure can be obtained, as shown in Fig. 6.

The power density-time curve shown in Fig. 6 is analyzed by the STFT method. The effect of extracting the high-frequency signal is the best when the sampling frequency is adjusted to $2500 \mathrm{~Hz}$ (the window width is $N=32$ ). The power spectral density-time curve and the waterfall of the power spectral density corresponding to the obtained power density are shown in Figs. 7 and 8, respectively.

The curve of the STFT coefficient at each time step corresponding to the frequency can be obtained by normalizing the power spectral density shown in Fig. 7. The main frequency and the STFT coefficient of power density for test point 4 at $t=2.43$ are shown in Fig. 9. 

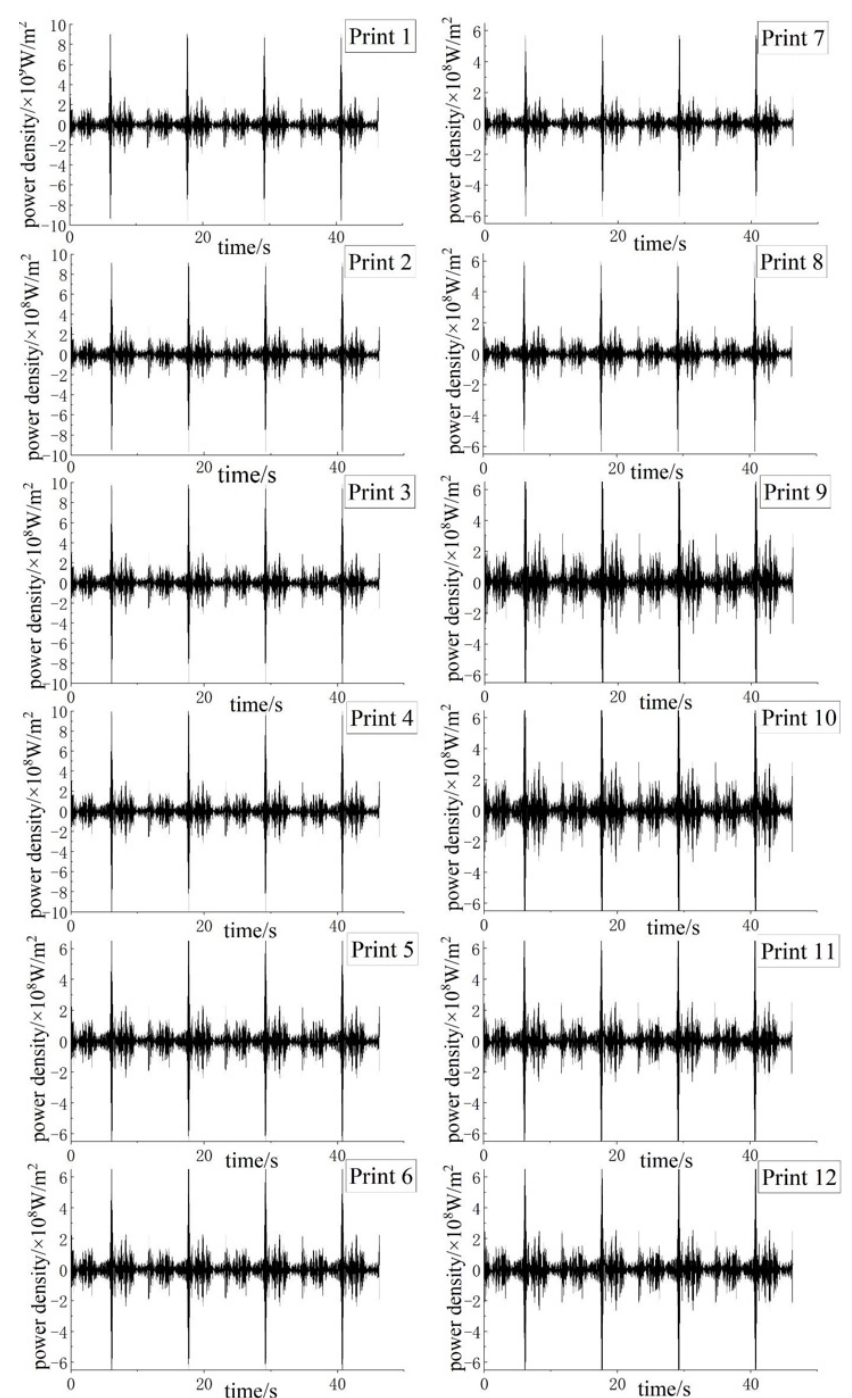

Fig. 6. Power density-time curve of each test point

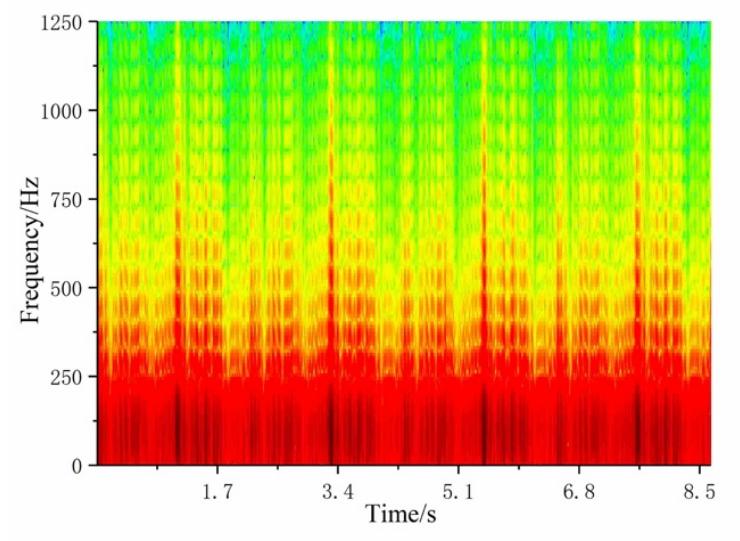

Fig. 7. Power spectral density

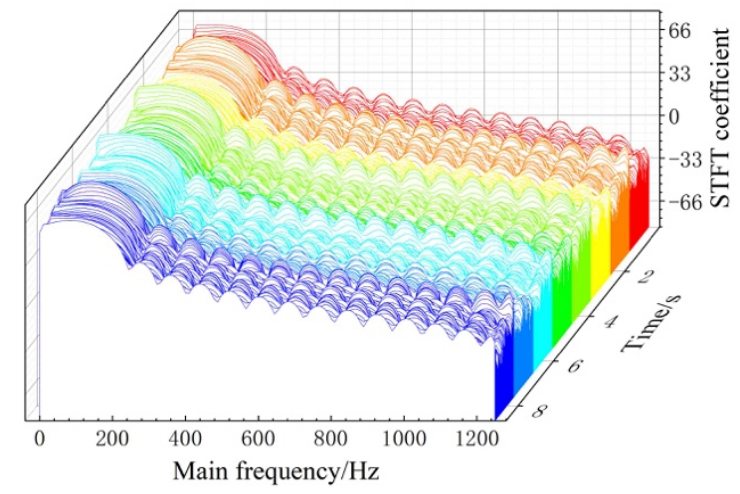

Fig. 8. Waterfall of power spectral density

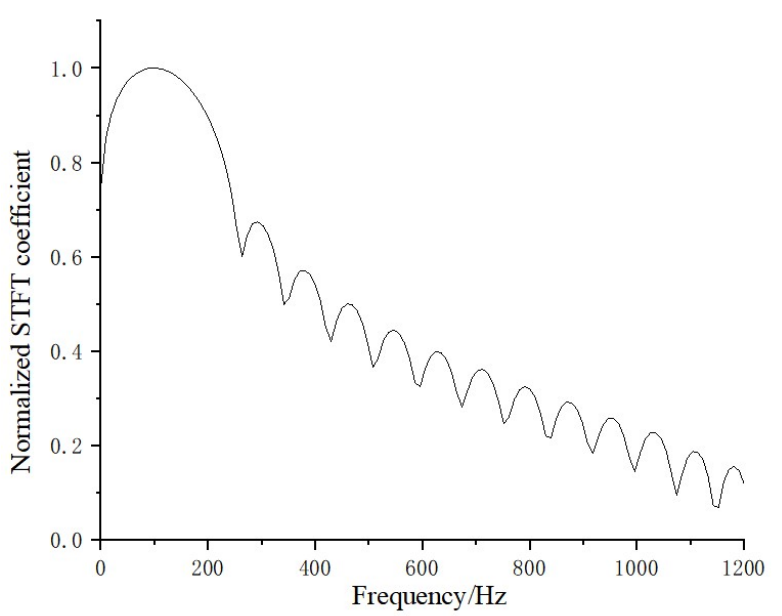

Fig. 9. Main frequency and STFT coefficient of power density at $t=2.43$

Fig. 9 shows that the power density includes 13 dominant frequencies at $t=2.43$, and the coordinates of the corresponding STFT coefficients can be expressed as follows: $\quad(87.89,0.9987) \quad, \quad(292.96,0.6740)$, $(380.85,0.5711),(458.98,0.5006),(546.87,0.4448)$, $(625,0.3989),(712.89,0.3612), \quad(791.01,0.3247)$, $(869.14,0.2921),(947.26,0.2577),(1025.3,0.2273)$, $(1103.5,0.1873)$, and $(1181.6,0.1560)$. The value of the power density at this time can be decomposed according to equation (7):

$(d S / d t)_{t=2.43}=A\left[\begin{array}{l}0.9987 \cos (87.89 \cdot \Delta t)+0.6740 \cos (292.96 \cdot \Delta t) \\ +0.5711 \cos (380.85 \cdot \Delta t)+0.5006 \cos (458.98 \cdot \Delta t) \\ +0.4448 \cos (546.87 \cdot \Delta t)+0.3989 \cos (625.00 \cdot \Delta t) \\ +0.3612 \cos (712.89 \cdot \Delta t)+0.3247 \cos (791.01 \cdot \Delta t) \\ +0.2921 \cos (869.14 \cdot \Delta t)+0.2577 \cos (947.26 \cdot \Delta t) \\ +0.2273 \cos (1025.3 \cdot \Delta t)+0.1837 \cos (1103.5 \cdot \Delta t) \\ +0.1560 \cos (1181.6 \cdot \Delta t)\end{array}\right]$

where $A$ is the amplitude of the power density with a time step of $\Delta t=0.0004 \mathrm{~s}$ at $t=2.43 \mathrm{~s}$. In accordance with Fig. 7, $(d S / d t)_{t=2.43}=1.75 \times 10^{7}, A=3.244 \times 10^{6}$ can be obtained. The damage rate corresponding to the main frequency at $t=2.43 \mathrm{~s}$ can be obtained according to equation (8), as shown in Table 2. 
Table 2. Damage rate of test point 4 in the interval of $0.0004 \mathrm{~s}$ at $t=2.43 \mathrm{~s}$

\begin{tabular}{l|l|l|l|l|l}
\hline Main frequency/Hz & Power density/ W/m & Cumulative cycles & Limit cycles & Damage rate & $\begin{array}{l}\text { Total damage rate } \\
\text { in }\end{array}$ \\
\hline 87.89 & $3.23 \times 10^{6}$ & 0.3516 & $3.16 \times 10^{10}$ & $1.11 \times 10^{-10}$ & \\
292.96 & $2.18 \times 10^{6}$ & 0.1172 & $1.91 \times 10^{10}$ & $6.13 \times 10^{-11}$ & \\
380.85 & $1.85 \times 10^{6}$ & 0.1523 & $2.07 \times 10^{10}$ & $7.38 \times 10^{-11}$ & \\
458.98 & $1.62 \times 10^{6}$ & 0.1835 & $2.18 \times 10^{10}$ & $8.43 \times 10^{-11}$ & \\
546.87 & $1.44 \times 10^{6}$ & 0.2187 & $2.27 \times 10^{10}$ & $9.64 \times 10^{-11}$ & \\
625.00 & $1.29 \times 10^{6}$ & 0.2500 & $2.38 \times 10^{10}$ & $1.06 \times 10^{-10}$ & $1.54 \times 10^{-9}$ \\
712.89 & $1.17 \times 10^{6}$ & 0.2887 & $2.42 \times 10^{10}$ & $1.2 \times 10^{-10}$ & \\
791.01 & $1.05 \times 10^{6}$ & 0.3164 & $2.48 \times 10^{10}$ & $1.27 \times 10^{-10}$ & \\
869.14 & $9.46 \times 10^{5}$ & 0.3476 & $2.54 \times 10^{10}$ & $1.37 \times 10^{-10}$ & \\
947.26 & $8.34 \times 10^{5}$ & 0.3789 & $2.61 \times 10^{10}$ & $1.45 \times 10^{-10}$ & \\
1025.30 & $7.36 \times 10^{5}$ & 0.4101 & $2.67 \times 10^{10}$ & $1.53 \times 10^{-10}$ & $1.61 \times 10^{-10}$ \\
1103.50 & $6.06 \times 10^{5}$ & 0.4414 & $2.75 \times 10^{10}$ & $1.67 \times 10^{-10}$ & \\
1181.60 & $5.05 \times 10^{5}$ & 0.4726 & $2.82 \times 10^{10}$ & \\
\hline
\end{tabular}

\subsection{Prediction of fatigue life}

Through this method, the damage within the interval of $\Delta t$ corresponding to each time step in the test can be calculated one by one. The cumulative fatigue damage rate of test point 4 on the hinged support structure can be obtained as
$2.30 \times 10^{-5}$ by adding the damage. According to equation (11), the fatigue life is $554.58 \mathrm{~h}$.

According to the same method, the damage of the 11 other test points in the test process is calculated, and the corresponding fatigue life is predicted. The damage and fatigue life of the 12 test points are shown in Table 3.

Table 3. Cumulative damage and fatigue life of each test point

\begin{tabular}{l|l|l|l|l|l}
\hline Test point & Cumulative damage & Prediction of fatigue life $/ \mathbf{h}$ & Test point & Cumulative damage & Prediction of fatigue life $/ \mathbf{h}$ \\
\hline 1 & $2.08 \times 10^{-5}$ & 611.80 & 7 & $1.32 \times 10^{-5}$ & 970.09 \\
2 & $2.11 \times 10^{-5}$ & 604.80 & 8 & $1.38 \times 10^{-5}$ & 926.19 \\
3 & $2.26 \times 10^{-5}$ & 564.95 & 9 & $2.42 \times 10^{-5}$ & 528.26 \\
4 & $2.30 \times 10^{-5}$ & 554.58 & 10 & $2.41 \times 10^{-5}$ & 529.58 \\
5 & $1.732 \times 10^{-5}$ & 737.72 & 11 & $1.91 \times 10^{-5}$ & 669.23 \\
6 & $1.72 \times 10^{-5}$ & 742.90 & 12 & $1.92 \times 10^{-5}$ & 665.03 \\
\hline
\end{tabular}

According to the actual investigation, no significant fatigue failure is found when the hinged support structure is used in the initial stage. The hinged support structure operates $10-15 \mathrm{~h}$ a day on average and is in the load-bearing state. The designed working life of the structure is 2 million sorties that of the pantograph, that is, the working life of the continuous lifting force of the pantograph is approximately $833 \mathrm{~h}$. The results of the fatigue analysis based on power density in this study are consistent with the actual work.

\section{Conclusions}

To analyze the fatigue life of a hinged support structure for an overhead conductor rail, this study proposed a novel method based on power density theory. The fatigue life of the hinged support structure was predicted according to the test of the collected dynamic stress. The following conclusions could be drawn:

1) The minimum fatigue life of the part where the elastic clamp is located is $554.58 \mathrm{~h}$, that of the part where the insulator and the connecting plate of the elastic clamp are connected is $737.72 \mathrm{~h}$, and that of the rotating base is 528.26 h.
2) The fatigue life of each part of the hinged support structure is close to the actual working life, thereby indicating that the fatigue life predicted by the proposed method is close to reality and has high reference value.

This study combined indoor experiments with theoretical research and proposed a novel method of fatigue analysis, which was applied to the fatigue analysis of the hinged support structure for an overhead conductor rail. The predicted fatigue life is close to reality. Hence, the results of this work may serve as a reference for the study of the fatigue life of overhead conductor rails. In the future, due to the lack of monitoring data on the actual working conditions of overhead conductor rails, field conditions and indoor experiments will be combined, the fatigue analysis method will be revised, and the understanding of the fatigue life of overhead conductor rails will be enhanced.

\section{Acknowledgements}

This work was supported by the National Key R\&D Program of China (2017YFB1201202).

This is an Open Access article distributed under the terms of the Creative Commons Attribution License 


\section{References}

1. Guan, J. F., Wu, J. Q., Fang, Y., "Review and prospect of research on overhead conductor rail". Urban Rapid Rail Transit, 29(06), 2016, pp.37-43.

2. Song, Y., Liu, Z. G., Wang, H. R., Lu, X. B., Han, Z. W., Zhang, j., "Analysis on influence of stochastic wind field on wind vibration fatigue of high-speed railway catenary". Journal of the China Railway Society, 37(07), 2015, pp.20-26.

3. Zhang, H. B., "Research on fatigue characteristics of $A C 25 \mathrm{kV}$ overhead rigid catenary cantilever support device". Master thesis of Southwest Jiaotong University, China, 2019, pp.1-55.

4. Qi, G. F., Xiao, X. H., Zhao, H., Chen, J. F., "Fatigue life evaluation on key components of high-speed railway catenary system". Journal of Central South University, 25(08), 2018, pp.1958-1965.

5. Massat, J. P., Nguyen Tajan, T. M. L., Maitournam, H., Balmès, E., "Fatigue analysis of catenary contact wires for high speed trains". In: 9 th World Congress on Railway Research, Lille, France: Science Arts \& Métiers, 2011, pp.1-11.

6. Ritzberger, D., Talic, E., Schirrer, A., "Efficient simulation of railway pantograph/catenary interaction using pantograph-fixed coordinates". International Federation of Automatic Control PapersOnLine, 48(1), 2015, pp.61-66.

7. Bucca, G., Collina, A., "Electromechanical interaction between carbon-based pantograph strip and copper contact wire: A heuristic wear model". Tribology International, 92, 2015, pp.47-56.

8. Kim, Y., Lee, K., Cho, Y., Guo, Z., Koo, J., Seok, C., "Fatigue safety evaluation of newly developed contact wire for eco-friendly high speed electric railway system considering wear". International Journal of Precision Engineering and Manufacturing-Green Technology, 3(4) 2016, pp.353-358.

9. Kim, Y., Lee, K., Li, H., Seok, C., Koo, J., Kwon, S., Cho, Y. H., "Fatigue life prediction method for contact wire using maximum local stress". Journal of Mechanical Science and Technology, 29(1), 2015, pp. $67-70$

10. Van, O. V., Massat, J., Balmes, E., "Waves, modes and properties with a major impact on dynamic pantograph-catenary interaction". Journal of Sound and Vibration, 402, 2017, pp.51-69.

11. Qi, G. F., Chen, J. F., Xiao, X. H., Song, Y. C., Ma, L., "Fatigue Load Spectrum of Key Parts of High-speed Railway Catenary". Journal of the China Railway Society, 37(10), 2015, pp.48-53.
12. Gregori, S., Tur, M., Nadal, E., Aguado, J. V., Chinesta, F., "Fast simulation of the pantograph-catenary dynamic interaction". Finite Elements in Analysis \& Design, 129, 2017, pp.1-13.

13. Tan, D. Q., Mo, J. L., Luo, J., Zhang, Q., Zhu, Z. R., "Failure Analysis of Positioning Hook and Support for High Speed Railway Catenary". China Railway Science, 39(05), 2018, pp.111-118.

14. Tan, D. Q., Mo, J. L., Peng, J. F., Luo, J., Chen, W. R., Zhu, M. h., "Research and Prospect on High-Speed Catenary Component Failure". Journal of Southwest Jiaotong University, 53(03), 2018, pp.610-619.

15. Zhao, H., "Fatigue life analysis of high-speed railway catenary dropper". Master thesis of Wuhan University, China, 2018, pp.1-61.

16. Chen, L. M., Peng, P. H., He, F., "Fatigue life analysis of dropper used in pantograph-catenary system of high-speed railway". Advances in Mechanical Engineering, 10(5), 2018, pp.1-10.

17. Zhang, J. W., Wu, J. Q., Chen, W. R., Guan, J. F., Zhong, Y., Xu, K. J., "Simulation Method for Dropper Dynamic Load Considering Horizontal Vibration Behaviour". International Journal of Simulation Modeling, 18(4), 2019, pp.620-631.

18. Wei, X. K., Meng, H. F., He, J. H., Jia, L. M., Li, Z. G., "Wear analysis and prediction of rigid catenary contact wire and pantograph strip for railway system". Wear, 442-443, 2020, pp.203118.

19. Liao, D., Zhu, S. P., Radicchi, F., Correia, J. A. F. O., De Jesus, A. M. P., Berto, F., "Recent advances on notch effects in metal fatigue: A review". Fatigue \& Fracture of Engineering Materials \& Structures, 43(4), 2020, pp.637-659.

20. Santecchia, E., Hamouda, A. M. S., Musharavati, F., Zalnezhad, E., Cabibbo, M., Mehtedi, M. E., Spigarelli, S., Eisenlohr, P., “A Review on Fatigue Life Prediction Methods for Metals". Advances in Materials Science and Engineering, 2016, 2016, pp.1-26.

21. Hrabowski, J., Ummenhofer, T., "Low cycle fatigue of welded very and ultra-high strength steels". Procedia Structural Integrity, 19, 2019, pp.259-266.

22. Newman, M. E. J., "Finding community structure in networks using the eigenvectors of matrices". Physical Review E, 74(3), 2006, pp.036104.

23. Fricke, W., Remes, H., Feltz, O., Lillemäe, I., Tchuindjang, D., Reinert, T., Nevierov, A., Sichermann, W., Brinkmann, M., Kontkanen, T., Bohlmann, B., Molter, L., "Fatigue strength of laser-welded thinplate ship structures based on nominal and structural hot-spot stress approach". Ships and Offshore Structures, 10(1), 2015, pp.39-44. 